\title{
Does the human immunodeficiency virus influence the vestibulocollic reflex pathways? A comparative study
}

\author{
Corresponding author: Barbara $M$. Heinze, $M(E C l)$ \\ Department of Speech-Language Pathology and Audiology, University of Pretoria, Pretoria, South Africa \\ Postal address: Department of Speech-Language Pathology and Audiology, Private Bag X20, Hatfield, Pretoria, \\ South Africa, 0028 \\ Email: barbara.heinze@up.ac.za \\ Fax: +27124203517
}

Tel: +27 124205358

\section{Bart M. Vinck, PhD}

Professor and Head of Department, Department of Speech-Language Pathology and Audiology, University of Pretoria, Pretoria, South Africa;

Email: bart.vinck@up.ac.za

\section{De Wet Swanepoel, PhD}

- Associate Professor, Department of Speech-Language Pathology and Audiology, University of Pretoria, Pretoria, South Africa;

- Ear Sciences Centre, School of Surgery, University of Western Australia, Nedlands, Australia;

- Ear Science Institute Australia, Subiaco, Australia.

Email: dewet.swanepoel@up.ac.za

\begin{abstract}
:
Background: This study compared vestibulocollic reflex (VCR) and vestibulo-ocular reflex (VOR) functioning in subjects with and without the human immunodeficiency virus (HIV). It also described test results throughout progression of the disease and compared it in HIV positive subjects with and without antiretroviral (ARV) therapies.

Methods: Subjects comprised 53 adults with HIV (mean age $38.5 \pm 4.4$ ) and 38 without HIV (mean age 36.9 \pm 8.2 ). Clinical examinations included cervical vestibular evoked myogenic potentials (cVEMPs) and bithermal caloric tests.
\end{abstract}


Results: Abnormal cVEMP and caloric results were significantly higher in the HIV positive group $(p=.001)$, with an odds ratio of 10.2. VCR and VOR involvement increased with progression of the disease. There were more abnormal test results in subjects using ARV therapies (66.7\%) compared to those not using ARV therapies (63.6\%), but this difference was insignificant. Conclusion: HIV seems to influence VCR pathways. Combining cVEMP and caloric tests may be useful to detect early neurologic involvement in HIV positive subjects.

\section{Key words:}

Human immunodeficiency virus

Vestibulocollic reflex

Vestibulo-ocular reflex

Vestibular evoked myogenic potentials

\section{Introduction}

The vestibular system senses movement and sends this information to the cerebellum and vestibular nuclei in the brainstem. Motion and other sensory information get processed and integrated, to stabilize gaze during head movement by means of the vestibulo-ocular reflex (VOR), and to maintain body and head stability by means of the vestibulospinal reflex (VSR) and vestibulocollic reflex (VCR) respectively. ${ }^{1,2}$ A pathway that includes the saccule, inferior vestibular nerve and vestibulospinal tract, mediates and actives the VCR. ${ }^{3,4}$ Cervical or collic vestibular evoked myogenic potentials (cVEMPs) are a manifestation arising from the VCR of the vestibulospinal tract ${ }^{5}$ and is mediated ipsilaterally by a three neuron arc. Uchino and colleagues $^{6}$ described the anatomic pathway of the VCR, also known as the sacculocollic reflex. Primary vestibular neurons that travel from the saccule via the inferior vestibular nerve, project into second order vestibular neurons in the lateral vestibular nucleus in the brainstem. From there, neurons descend in the medial vestibulospinal tracts and connect to the motor nuclei of 
the accessory nerve. Third order vestibular neurons descend to the flexor and extensor neck muscles via the medial vestibulospinal tract. ${ }^{6}$

cVEMPs are ipsilaterally evoked short latency responses measured with an active electrode over a contracted sternocleidomastoid (SCM) muscle ${ }^{7}$, capable of evoking the $\mathrm{VCR}^{8}$ and is perhaps the most direct way of testing VCR functioning. ${ }^{9}$ Therefore, abnormalities of the cVEMP may indicate a lesion at any point along the VCR pathway.

Testing of the VOR pathways, which include the horizontal semicircular canal, superior vestibular nerve and ascending neural path to the extra-ocular muscles ${ }^{10}$, is well characterized and the basis of many commonly used vestibular tests. ${ }^{2}$ These include, but are not limited to the caloric test ${ }^{11}$ and rotational tests. ${ }^{12}$ Testing of the VSR pathways may include posturography. ${ }^{11}$

The VOR and VSR pathways have been examined and described among individuals with the human immunodeficiency virus (HIV) and acquired immunodeficiency syndrome (AIDS). A recent systematic literature review summarized all vestibular tests and findings in subjects with HIVIAIDS demonstrating that tests of vestibular function concentrated on the VOR and VSR pathways only. ${ }^{13}$ To date no studies have investigated the VCR pathways, as tested with cVEMPs, in subjects with HIVIAIDS. This lack of information may in apart be attributable to that fact that cVEMPs have only recently been included as part of clinical test-batteries for vestibular function having proved useful in identifying vestibular disorders. ${ }^{9}$ Therefore, this study aimed i) to describe and compare the functioning of the VCR with the well characterized VOR in subjects with and without HIV, ii) to describe the VCR and VOR throughout progression of the disease, and iii) to compare the VCR and VOR in HIV positive subjects receiving ARV therapies to those who were not receiving ARV therapies. 


\section{Materials and methods}

The Research and Ethics Review Committee of the University of Pretoria and a tertiary referral hospital approved the current study. A cross-sectional comparative research design was employed and the method of convenience sampling was used to recruit subjects. Each subject provided written informed consent to participate in the study.

TABLE I

DESCRIPTION OF SUBJECTS

\begin{tabular}{lll}
\hline Description & HIV negative group & HIV positive group \\
\hline Number of subjects $(n)$ & 38 & 53 \\
Mean age & $36.9(\mathrm{SD}=8.2)$ & $38.5(\mathrm{SD}=4.4)$ \\
Min-Max age & $20-50$ years & $23-49$ years \\
Gender distribution & Male $=47.4 \%(n=18)$ & Male $=55 \%(n=29)$ \\
& Female $=52.6 \%(n=20)$ & Female $=45 \%(n=24)$ \\
CDC categories & Not applicable & CDC category 1: $n=15$ \\
& & CDC category 2: $n=20$ \\
ARV therapy users & CDC category 3: $n=18$ \\
& Not applicable & ARV therapy users: $n=42$ \\
& & non-ARV therapy users: $n=11$
\end{tabular}

SD = Standard Deviation; CDC = Centres for Disease Control; ARV = antiretroviral

\section{Subjects}

Table I summarizes the description of participating subjects. Subjects with HIV formed part of the experimental group and were drafted from the Infectious Disease (ID) Clinic at a tertiary referral hospital in South Africa. The subjects' HIV status were confirmed by blood serological tests and this was documented in their medical records. The researchers obtained written informed consent to access their medical records containing this data. The subjects without HIV were employees of the tertiary referral hospital and acquaintances of the researchers who agreed to undergo a blood serological test for HIV. The subjects who were confirmed to be HIV negative formed part of the control group. A total of 91 subjects, 53 adults with HIV and 38 without HIV were evaluated for participation in the study. I summarizes the description of participating subjects. There were no statistically significant differences in mean ages between the groups $(p=0.26$; t-test). Literature demonstrated that age affected the vestibular system after 
55 to 65 years, ${ }^{14}$ therefore, in order to minimize the likelihood of age affecting the results, only subjects below the age of 50 were allowed to participate in the study.

The subjects with HIV were further divided into categories according to their cluster of differentiation $4(\mathrm{CD} 4+)$ cell counts as documented in their medical files at the ID clinic at the time of participation in the study. Subjects with counts higher than 500 cells/uL were assigned to the Centre for Disease Control and Prevention (CDC) (CDC, 1993) category 1, while those with counts of 200-499 cells/uL and less than 200 cells/uL were assigned to CDC categories 2 and 3 respectively. HIV positive subjects were evenly distributed between the three CDC categories. Fifteen subjects were in category 1 (eight male, seven female), 20 subjects in category 2 (eight male, 12 female) and 18 subjects in category 3 (eight male, 10 female).

The subjects with HIV were also divided into two other groups, namely those who received ARV therapies $(n=42)$ and those who were not receiving ARV therapies $(n=11)$. The subjects who were exposed to ARV therapies received at least three of the following drug combinations: tenofovir, lamivudine, efavirenz, emtricitabine, nevirapine, stavudine, zidovudine and lopinavir/ritonavir.

\section{Otologic and audiological examination}

An otoscopic examination was performed to inspect the external auditory canal for any, debris or foreign objects that might cause occlusion of the ear canal and to identify any possible perforation of the tympanic membrane. Subjects with obstructed ear canals were referred to a clinician for extraction prior to participation in the study. Tympanometry was performed using a diagnostic Y-226 Hz probe tone (GSI Tympstar, Grason-Stadler). The following criteria ${ }^{15}$ were used for normal adult admittance profiling: ear canal volume (0.8 to $2.0 \mathrm{ml})$, compliance $(0.3$ to $1.8 \mathrm{ml})$ and middle ear pressure (-100daPa to $+50 \mathrm{daPa})$. Type A tympanograms were revealed for 49 subjects with HIV and for 38 subjects without HIV. Pure tone audiometry (air and bone 
conduction) was performed to determine the presence of air-bone gaps (GSI 61, GrasonStadler). Air-bone gaps larger than $10 \mathrm{~dB}$ in the four HIV positive subjects with abnormal compliance and middle ear pressure were found, suggesting a conductive component. The 49 HIV positive and 38 HIV negative subjects with type A tympanograms showed no air-bone gaps.

\section{VOR test}

Caloric tests were used to describe the VOR. Rotational testing was not available to the researchers. Tests of spontaneous nystagmus, with and without fixation, preceded caloric stimulation. Video nystagmography (Visual Eyes infrared video-based system from Micromedical Technologies Inc.) was used to record any spontaneous nystagmus and caloric induced nystagmus. Bithermal $\left(\mathrm{cool} 24^{\circ} \mathrm{C}\right.$, warm $47^{\circ} \mathrm{C}$ ) air caloric testing (AirFX, Micromedical Technologies Inc.) was used to irrigate the external auditory canal. Air caloric was chosen over water irrigation, because water irrigation of the external ear canal may result in damage to the delicate skin lining of the outer ear, which in turn places it at risk for invasive external otitis due to bacterial invasion. ${ }^{16}$ This frequently occurs in those who are immunocompromised, such as persons with HIV. Subjects were placed in a supine position with the head tilted forward at an angle of $30^{\circ}$ from the horizontal plane for correct positioning of the horizontal semicircular canals. Air was irrigated for 60 seconds and the time elapsing between stimuli was five minutes. The peak of the slow phase eye velocity (SPV) of caloric nystagmus post-irrigation was used as a parameter of superior vestibular nerve and horizontal canal function. Jongkees' formula ${ }^{17}$ was used to calculate unilateral weakness or asymmetry and directional preponderance. A unilateral weakness or asymmetry of $\geq 20 \%,{ }^{11,18}$ directional preponderance of $\geq 30 \%$, ${ }^{11}$ bilateral weakness and hyperreflexia was considered abnormal. Bilateral weakness or hypoactive responses were regarded as the total warm responses from both sides, less than 11 degrees per second, and the total cool responses from both sides less than 6 degrees per second. ${ }^{19}$ Hyperreflexia or hyperactive responses were regarded as total responses from both sides of more than 221 degrees per second. ${ }^{18}$ 


\section{VCR test}

The cVEMP procedure was performed using an auditory evoked potential system (Biologic Navigator Pro, Natus Medical Incorporated). Subjects were comfortably seated with the head rotated approximately $45^{\circ}$ to the opposite side of the ear being tested ear. A blood pressure manometer with a rolled up inflatable cuff positioned between the subject's hand and jaw was used as feedback method of the contracted sternocleidomastoid (SCM) muscle during the recording of the cVEMP. The subjects pushed with their heads against the rolled up inflatable cuff and were asked to sustain a pressure of $40 \mathrm{mmHg}$. This allowed control of the SCM contractions and ensured comparable muscle contractions between the left and right side..$^{20}$ Both the subjects and the investigator monitored this sustained pressure. Insert-type earphones (Etymotic-ER-3) with disposable foam tips were used. Every measurement, including absent responses, was repeated twice to test for wave reproducibility and to eliminate potential artifacts. The average of the two recordings was used for analysis. The first peak on the waveforms was marked as $\mathrm{P} 1$, while the second was marked as $\mathrm{N} 1$ within a period of 30 milliseconds (ms).

The researchers recorded and measured the latencies of P1 and $\mathrm{N} 1$ in ms, inter-peak amplitude in microvolt $(\mu \mathrm{V})$, and amplitude asymmetry in percentage (\%). The asymmetry ratio was determined by calculating the interaural amplitude difference according to the following formula where $A_{L}$ indicated the amplitude for the left ear and $A_{R}$ the amplitude for the right ear: $\left[\left(A_{L}-\right.\right.$ $\left.\left.A_{R}\right) /\left(A_{L}+A_{R}\right)\right] \times 100$. Responses were interpreted as follows: (i) the absence of unilateral or bilateral waveforms were considered abnormal (absence of an identifiable P1 and N1); (ii) two standard deviations above the mean of the HIV negative group were used to calculate the upper limits for P1 and N1 latencies (17.0ms and 26.3ms respectively). Latencies above these upper limits were regarded as present yet delayed, and considered abnormal; and (iii) the presence of 
an amplitude asymmetry ratio of $\geq 40 \%$ was considered abnormal, since it indicated side-to-side differences in amplitude. ${ }^{21}$

\section{Results and analysis}

All analyses of data were performed using the statistical software package SPSS for Windows version 21. Means, standard deviations ( \pm ) and percentages were used to describe the data. One-way analysis of variance (ANOVA) was used to compare the distribution of HIV positive subjects between the three CDC categories. The One-Sample Kolmogorov-Smirnov Test was used to demonstrate normality of data. The Independent Samples t-Test was used to compare mean values between the experimental and control groups. $P$ values $<0.05$ were accepted as statistically significant. Odds ratios were calculated. The chi-square non-parametric test was used to compare the findings between the two study groups and the three CDC categories.

\section{cVEMP and caloric test abnormalities}

Abnormal cVEMP and caloric test results were found in $66 \%(n=35)$ of the subjects with HIV, compared to only $15.8 \%(n=6)$ of the subjects without HIV, indicating a significantly higher occurrence of pathology in subjects with HIV $(p=.001$; chi-square). Four absent cVEMP recordings were from the four HIV positive subjects with abnormal tympanograms and air-bone gaps. This association between vestibular signs and HIV was further confirmed by the odds ratio. An odds ratio of 10.2 was obtained, showing a 10.2 times higher risk for showing abnormal cVEMP and caloric responses in persons who are HIV positive.

Table II shows the distribution of abnormal cVEMP and caloric test results in the HIV positive and HIV negative groups. Abnormal cVEMP results were found in $43.4 \%(n=23)$ and abnormal caloric results in $35.8 \%(n=19)$ of subjects with HIV. Abnormal cVEMP results due to middle ear pathology were found in $7.5 \%(n=4)$ subjects with HIV. The cVEMP results were abnormal in $10.5 \%(n=4)$ and the caloric results were abnormal in $10.5 \%(n=4)$ of the subjects without HIV. 
TABLE II

DISTRIBUTION OF ABNORMAL cVEMP AND CALORIC RESULTS IN THE TWO STUDY GROUPS

\begin{tabular}{ccc} 
HIV positive group & HIV negative group & $\boldsymbol{p}$ value \\
\hline $\mathbf{n}(\%)$ & $\mathbf{n}(\%)$ \\
\hline $12(22.6)$ & $2(5.3)$ \\
$8(15.1)$ & $2(5.3)$ \\
$11(20.8)$ & $2(5.3)$ \\
$4(7.5)$ & 0 & \\
$35(66)$ & $6(15.8)$ & .001
\end{tabular}

Abnormal cVEMP

Abnormal caloric

Abnormal cVEMP with abnormal caloric

Abnormal cVEMP due to MEP

Total abnormalities

MEP = middle ear pathology, as demonstrated by abnormal middle ear compliance and pressure, and air-bone gaps

Table III indicates the occurrence of abnormal cVEMP results according to absent waveforms, delayed $\mathrm{P} 1$ and/or $\mathrm{N} 1$ latencies and amplitude asymmetry $\geq 40 \%$. In the HIV positive group,

TABLE III

DESCRIPTION OF ABNORMAL CVEMP AND CALORIC TEST FINDINGS IN THE HIV POSITIVE AND HIV NEGATIVE SUBJECTS

\begin{tabular}{lcc}
\hline & & \\
Abnormal parameters & HIV positive group & HIV negative group \\
\cline { 2 - 3 } & $\mathbf{n}(\%)$ & $\mathbf{n}(\%)$ \\
\hline cVEMP & $3(5.7)$ & $1(2.6)$ \\
Absent* unilateral & $5(9.4)$ & $1(2.6)$ \\
Absent bilateral & $1(1.9)$ & - \\
Delayed unilateral & $5(9.4)$ & - \\
Delayed bilateral & $3(5.7)$ & - \\
Absent* unilateral with delayed unilateral & $10(18.9)$ & $3(7.9)$ \\
Asymmetry ratio $\geq 40 \%$ & $15(28.3)$ & $3(7.9)$ \\
Caloric & $1(1.9)$ & $1(2.6)$ \\
Unilateral weakness & $2(3.8)$ & - \\
Bilateral weakness & $3(5.7)$ & - \\
Hyperreflexia & & \\
Directional preponderance & & \\
\hline
\end{tabular}

${ }^{*}$ data of the four subjects with middle ear pathology excluded here

$20.8 \%(n=11)$ of subjects showed absent cVEMP recordings, not including the four subjects with middle ear pathology. Of the six subjects with a unilaterally absent cVEMP, five had an absent cVEMP on the left side, and one had an absent cVEMP on the right side. Only one subject in the HIV negative group showed absent cVEMPs bilaterally. There was a significantly higher occurrence of absent cVEMPs among the subjects with HIV than for subjects without HIV $(p=.003$; chi-square). Table III further indicates that in the HIV positive group $17 \%(n=9)$ of 
subjects presented with delayed latencies. Four subjects showed delayed latencies unilaterally and five bilaterally.

There was no significant difference observed regarding mean latencies of P1 and N1 to the left or right side in either of the study groups ( $p>0.05$; t-test). Table IV indicates the distribution of mean P1 and N1 latencies, as well as inter-peak amplitude differences in both the HIV positive and negative groups as recorded from the cVEMP test. P1 latencies were significantly delayed statistically (but not clinically), in the HIV positive group. N1 latencies showed no difference between the two groups.

\section{TABLE IV}

MEAN LATENCY AND INTER-PEAK AMPLITUDE RESULTS IN THE HIV POSITIVE AND HIV NEGATIVE GROUPS FROM CVEMP RECORDINGS

\begin{tabular}{ccccc}
\hline & $\mathrm{n}$ & $\mathrm{P} 1$ latency $(\mathrm{ms})$ & $\mathrm{N} 1$ latency $(\mathrm{ms})$ & I-P amplitude $(\mu \mathrm{V})$ \\
\hline & & & & \\
HIV positive group & 84 & $15.2 \pm 2.2$ & $21.7 \pm 2.4$ & $201.2 \pm 51.1$ \\
HIV negative group & 73 & $13.9 \pm 1.6$ & $21.7 \pm 4.1$ & $172.7 \pm 63.4$ \\
p value* (t-test) & & .001 & .89 & .003 \\
\hline
\end{tabular}

$\bar{N}=$ number of ears (data not used for ears with absent cVEMP); \pm = standard deviation; I-P = inter-peak.

cVEMP and caloric test results throughout disease progression

Figure 1 illustrates the cVEMP and caloric test results throughout the progression of the disease. The HIV positive subjects were divided into the three CDC (1993) categories based upon their CD4+ cell counts at the time of participation. Normal cVEMP and caloric test results were recorded in $34 \%$ of subjects with HIV. The occurrence of abnormal test results increased from $13.3 \%$ in category 1 , to $22.6 \%$ in category 2 and $30.1 \%$ in category 3 of disease progression (Figure 1). 


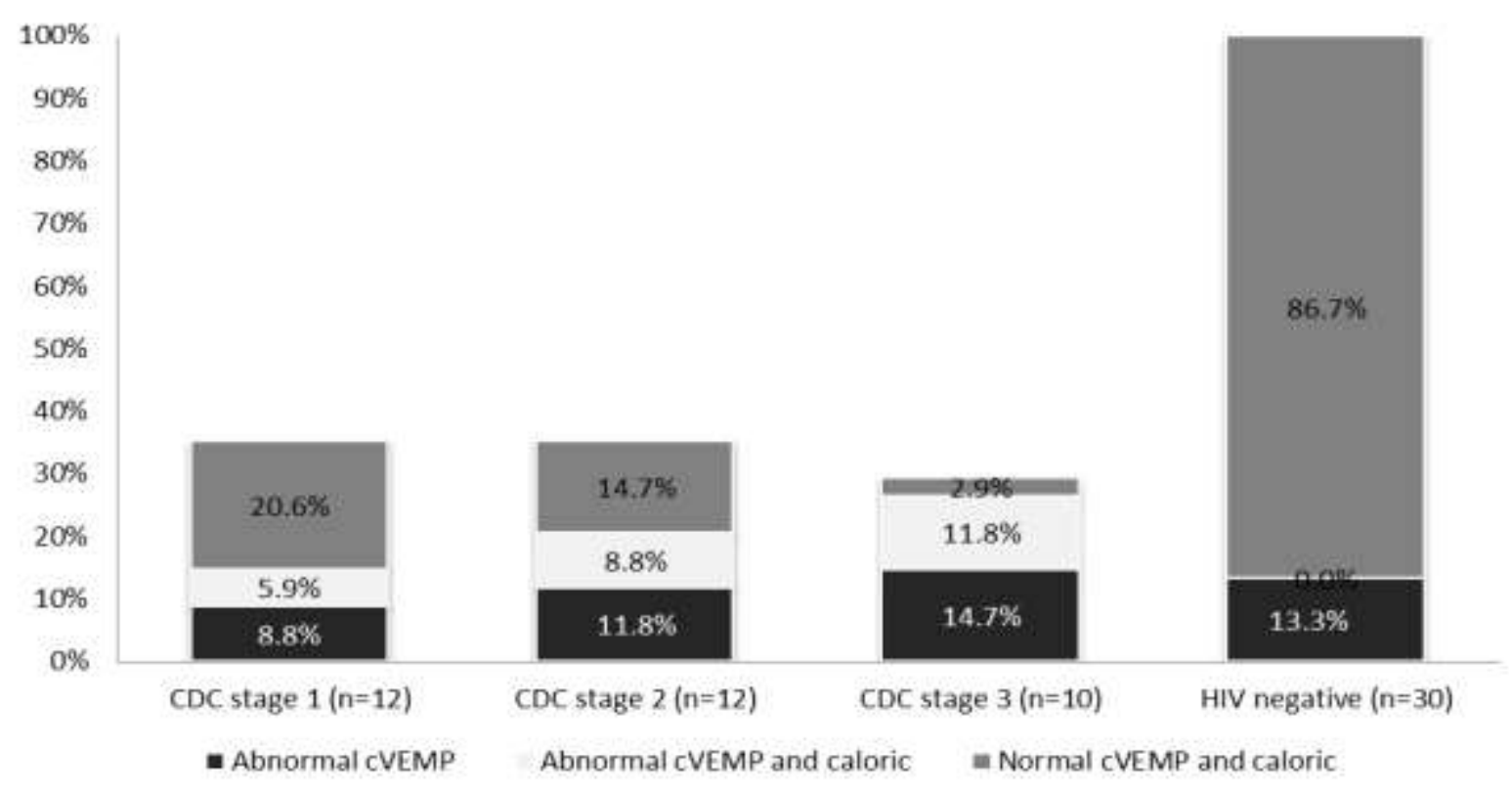

FIG. 1 cVEMP and caloric test results throughout disease progression

CVEMP and caloric test results of the HIV positive subjects receiving ARV therapies compared to those who were not receiving $A R V$ therapies

Table $V$ shows the distribution of abnormal cVEMP and caloric results of the HIV positive subjects receiving ARV therapies compared to those who were not receiving ARV therapies. Of the 42 ARV therapy users, $66.7 \%(n=28)$ showed abnormal cVEMP and caloric test results. Of the 11 non-ARV therapy users, $63.6 \%(n=7)$ showed abnormal cVEMP and caloric test results. Although the occurrence of abnormal test results were higher among the ARV therapy users, these difference were not statistically significant ( $p=.91$; chi-square)

TABLE V

DISTRIBUTION OF ABNORMAL CVEMP AND CALORIC RESULTS OF THE HIV POSITIVE SUBJECTS RECEIVING ARV THERAPIES COMPARED TO THOSE WHO WERE NOT RECEIVING ARV THERAPIES

Abnormal cVEMP

Abnormal caloric

Abnormal cVEMP with abnormal caloric

Total abnormalities

\begin{tabular}{ccc} 
ARV users $(\mathrm{n}=42)$ & $\begin{array}{c}\text { Non-ARV } \\
\text { users }(n=11)\end{array}$ & $p$ value \\
\hline$n(\%)$ & $n(\%)$ \\
\hline
\end{tabular}

$13(30.9)$

$4(9.5)$

$11(26.2)$

$3(27.3)$

$4(36.4)$

$0(0)$

$7(63.6)$ .91

$\mathrm{ARV}=$ antiretroviral therapy 


\section{Discussion}

This study demonstrated that subjects with HIV presented with significantly more abnormal cVEMP and caloric test findings compared to those without HIV. Two-thirds of the subjects with HIV $(66 \%)$ presented with at least one abnormality on the cVEMP and caloric test parameters, compared to only $15.8 \%$ of subjects without HIV. The calculated odds ratio suggested that subjects with HIV had a 10.2 higher risk of presenting abnormal cVEMP and caloric test results than subjects without HIV.

Regarding the recording of reliable cVEMP responses, the use of a correction algorithm remains the favored method, as well as monitoring the electromyographic (EMG) activity of the SCM. ${ }^{20}$ The current study did not have access to these algorithms or EMG systems and, subsequently, this is a limitation of the study. However, results of a recent study concluded that the use of the blood pressure manometer may be a useful alternative in recording reliable CVEMP responses. ${ }^{20}$

The caloric findings in the current study could be compared with those of four previous research reports that employed a group study design of adult subjects with HIV ${ }^{22-25}$ The abnormal caloric test findings demonstrated the presence of abnormal functioning of the VOR pathway. Three of the four studies indicate similar abnormal caloric test findings in subjects with HIV compared to the current study $(26.5 \% ; n=9)$. The two most recent studies ${ }^{22,23}$ found abnormal caloric test results in $43.3 \%$ and $50 \%$ respectively in their sample of subjects with HIV. Their samples of subjects were all symptomatic, since they suffered from chronic dizziness. An earlier study found slightly less abnormal caloric test results compared to the current study, namely $11.6 \%$ in their sample of subjects with $\mathrm{HIV} .{ }^{25}$ In contrast, only one study reported no abnormalities in caloric test findings in their sample of subjects with $\mathrm{HIV} .{ }^{24}$ No noticeable differences were found between the caloric test protocols employed in these reports, but the subjects in the study by Castello and colleagues ${ }^{24}$ were all asymptomatic without any vestibular symptoms such as 
vertigo, dizziness or disequilibrium, which could explain the absence of abnormal caloric responses. It is interesting to note that $66.4 \%(n=29)$ of HIV positive subjects in the study by Hausler and colleagues ${ }^{25}$ were asymptomatic, yet the authors reported abnormal caloric test findings even among those subjects. However, these authors did not indicate whether their subjects used ARV therapies or any medication for secondary infections that could contribute to the abnormal caloric test findings.

To date, no other research reports have utilized cVEMP in subjects with HIV to describe the functioning of the VCR pathways. The current study demonstrated a significantly higher number of abnormal cVEMP results in subjects with HIV than in subjects without HIV. The mean P1 latencies were statistically significantly delayed in the HIV positive group; however, the differences were minimal. Such small differences would not be considered clinically significant and the mean $\mathrm{P} 1$ latency for the HIV positive group was well within normal clinical expectations for tone evoked cVEMP responses. The results of the abnormal cVEMP tests suggest a high occurrence of abnormal functioning of the VCR pathways in subjects with HIV. The results of the abnormal caloric test also suggest a higher occurrence of abnormal functioning of the VOR pathways in subjects with HIV opposed to those without the disease. The caloric test only assesses the lower frequency responses, namely at $0.003 \mathrm{~Hz} ;{ }^{26}$ the mid and higher frequencies are therefore not assessed, meaning that the VOR as assessed with the caloric test may be even higher.

Possible mechanisms for the increased VCR and VOR abnormalities may include opportunistic infections, ototoxic treatments and direct effects of HIV. ${ }^{13}$ Opportunistic infections may partly affect the functioning and integrity of both branches of the vestibular nerve (and the structures that they innervate). Young ${ }^{27}$ reported five patients with the herpes zoster virus, a common HIVrelated opportunistic infection, suffering from vertigo. Unfortunately, the author did not indicate in the report whether these subjects were HIV positive. Nonetheless, all five subjects (100\%) 
had absent cVEMPs. In addition, four subjects (80\%) had absent caloric responses. In another study, 10 subjects with Ramsay Hunt syndrome, also a common HIV related opportunistic infection, underwent cVEMP and caloric testing. ${ }^{28}$ Once again, the authors did not indicate the subjects' HIV status. Similar to Young, ${ }^{27}$ the study revealed abnormal cVEMP results in seven subjects $(70 \%)$ and abnormal caloric results in all 10 subjects $(100 \%)$. Another study ${ }^{29}$ demonstrated abnormal cVEMP $(23.1 \%)$ and caloric $(30.8 \%)$ test results in subjects infected with cytomegalovirus (HIV status of subjects were not indicated), also a common HIV related opportunistic infection that has been reported to cause sensorineural hearing loss and peripheral and central neurologic manifestations in subjects infected with HIV. ${ }^{30,31}$ These findings suggest that opportunistic infections like herpes zoster virus and Ramsay Hunt syndrome may result in involvement of both the VCR and VOR pathways.

The use of ARV therapies could also contribute to the higher occurrence of abnormal cVEMP and caloric test findings in subjects with HIV. Those exposed to ARV therapies presented with slightly more abnormalities $(66.7 \%)$ in cVEMP and caloric testing than those without ARV therapies (63.7\%); these differences, however, were not statistically significant. Recent studies found similar results with $A B R$ testing and demonstrated a higher occurrence of abnormal ABR findings in a group of subjects receiving ARV therapies (62.5\%) compared to those not receiving ARV therapies (50\%), although the difference was not statistically significant. ${ }^{32}$ Such findings suggest that the auditory and vestibular nerves, as well as the structures that they innervate, are at risk due to possible ototoxic effects of some ARV therapies. ARV regimes may consist of three or more classes of drugs, and one or more of these are nucleoside/nucleotide analog reverse transcriptase inhibitors (NRTIs). ${ }^{33}$ One adverse effect of NRTIs is mitochondrial toxicity, which is responsible for, among other, myopathy and neuropathy. ${ }^{34}$ Neuropathy is a dysfunction of the nervous system, and may therefore include the vestibular branches of the eighth cranial nerve. Additionally, there are case reports of ototoxic sensorineural hearing loss associated with the use of NRTIs which may have been induced by reduction in the 
mitochondrial DNA content, although ageing and the virus itself could have contributed to mitochondrial DNA mutations. ${ }^{35}$ Therefore, if these drugs may cause sensorineural hearing loss and affect the auditory brainstem pathways, it is also likely that they affect the vestibular nerves and/or end organs in subjects who received ARV therapies. A recent study compared the vestibular function of HIV positive subjects that used highly active antiretroviral therapy with age and gender-matched HIV negative subjects. ${ }^{36}$ They performed vestibular screening tests which consisted of head thrust tests, Dix-Hallpike maneuvers and Romberg balance tests and found no significant difference between the two groups. The subjects were excluded if they had vestibular complaints, which could suggest that they were centrally compensated. Therefore, the vestibular screening tests that were employed may have been underpowered for the detection of subclinical vestibular involvement in subjects who were centrally compensated.

The occurrence of abnormal cVEMP and caloric test findings increased throughout progression of the disease-from $13.3 \%$ in early stages (CDC category 1 ) to $30.1 \%$ in advanced stages (CDC category 3). Three previous studies, that also used a cross-sectional research design, demonstrated an increase in vestibular involvement from early to advanced stages of the disease. ${ }^{22,23,25}$ A detailed summary of their findings has been reviewed elsewhere. ${ }^{13}$ There is a higher occurrence of abnormal cVEMP and caloric test findings in the advanced stages of HIV, due to the reduction in CD4+ cell counts that places the infected individual at-risk for various opportunistic infections. This necessitates the use of ARV therapies to strengthen immunity in order to combat opportunistic infections; however, the vestibular nerves and structures of the vestibular end-organs may be susceptible to ototoxicity and also to the infections themselves, resulting in abnormal cVEMP and caloric test results.

The eighth cranial nerve and brainstem pathways may undergo neuropathologic changes such as subcortical demyelination because of the HIV infection itself. ${ }^{37,38}$ This may explain the abnormalities in ABR measured in HIV positive individuals with and without clinical features of 
the disease, irrespective of normal hearing thresholds. ${ }^{37}$ Since the ABR may detect subclinical pathologic changes in the peripheral auditory nervous system, cVEMP and caloric testing may detect pathologic changes in the VOR and VCR pathways respectively in adults infected with HIV. Posturography may also be useful in detecting pathologic changes in the VSR pathways, ${ }^{39}$ however this test procedure was unavailable to the researchers.

\section{Conclusion}

There was a significantly higher occurrence of abnormal cVEMP responses and caloric test results in the adults with HIV than in those without HIV. The abnormalities shown by the cVEMP and caloric tests were probably due to pathology of the VCR and VOR pathways respectively. A combination of cVEMP and caloric tests in the vestibular test battery for adults infected with HIV may offer a tool for detecting early neurologic involvement, irrespective of disease progression and clinical manifestations.

\section{Acknowledgements}

Declaration of interest: The authors report no conflicts of interest. The authors alone are responsible for the content and writing of the paper.

\section{References}

1. Goebel JA. Practical anatomy and physiology. In: Goebel JA, ed. Practical management of the dizzy patient. Second ed. Philadelphia; Baltimore; New York; London; Buenos Aires; Hong Kong; Sydney; Tokyo: Wolters Kluwer; 2008:1-13

2. Wilson VJ, Boyle R, Fukushima K, Rose PK, Shinoda Y, Suqiuchi Y, et al. The vestibulocollic reflex. J Vestib Res 1995;5:147-70 
3. Ito K, Ishimoto S-, Murofushi T. Narrow internal auditory meatus: An idiopathic case confirming the origin and pathway of vestibular evoked myogenic potentials in humans. Arch Otolaryngol Head Neck Surg 2001;127:275-8

4. Rosengren SM, Welgampola MS, Colebatch JG. Vestibular evoked myogenic potentials: Past, present and future. Clin Neurophysiol 2010;121:636-51

5. Uchino $Y$, Sato $H$, Sasaki M, Imaqawa M, Ikeqami H, Isu N, et al. Sacculocollic reflex arcs in cats. J Neurophysiol 1997;77:3003-12

6. Colebatch JG, Halmagyi GM, Skuse NF. Myogenic potentials generated by a click-evoked vestibulocollic reflex. J Neurol Neurosurg Psych 1994;57:190-7

7. Colebatch JG, Rothwell JC. Motor unit excitability changes mediating vestibulocollic reflexes in the sternocleidomastoid muscle. Clin Neurophysiol 2004;115:2567-73

8. Welgampola MS, Colebatch JG. Characteristics and clinical applications of vestibular-evoked myogenic potentials. Neurology 2005;64:1682-8

9. Wester M. Vestibular evoked myogenic potentials. In: Atcherson SR, Stoody TM, eds. Auditory electrophysiology: A clinical guide. New York, NY: Thieme Medical Publishers; 2012:289-306

10. Fife TD, ed. Overview of anatomy and physiology of the vestibular system. ; 2010; Handbook of Clinical Neurophysiology; No. 9

11. Baloh RW, Kerber KA. Clinical neurophysiology of the vestibular system. Fourth ed. Oxford: University Press; 2011

12. Brandt T, Strupp M. General vestibular testing. Clin Neurophysiol 2005;116:406-26 
13. Heinze BM, Swanepoel DW, Hofmeyr LM. Systematic review of vestibular disorders related to human immunodeficiency virus and acquired immunodeficiency syndrome. J Laryngol Otol $2011 ; 125: 881-90$

14. Maes L, Dhooge I, D'haenens W, Bockstael A, Keppler H, Philips B, et al. The effect of age on the sinusoidal harmonic acceleration test, pseudorandom rotation test, velocity step test, caloric test, and vestibular evoked myogenic potential test. Ear Hear 2010;31:84-94

15. Jerger J. Clinical experience with impedance audiometry. Arch Otolaryngol 1970;92:311-24

16. Zikk D, Rapoport Y, Himelfarb MZ. Invasive external otitis after removal of impacted cerumen by irrigation. N Engl J Med 1991;325:969-70

17. Jongkees LB, Maas J, Philipszoon A. Clinical nystagmography: a detailed study of electronystagmography in 341 patients with vertigo. Pract Otorhinolaryngol (Basel. 1962;24:65-93

18. Jacobson GP, Newman CW, Peterson EL. Interpretation and usefulness of caloric testing. In: Jacobson GP, Newman CW, Kartush JM, eds. Handbook of balance function testing. San Diego, London: Singular Publishing Group, Inc.; 1997:193-234

19. Barber HO, Stockwell CW. Manual of electronystagmography. Second ed. St Louis: C.V. Mosby; 1980

20. Maes L, Vinck BM, De Vel E, D'Haenens W, Bockstael A, Keppler H, et al. The vestibular evoked myogenic potential: A test-retest reliability study. Clin Neurophysio. 2009;120:594-600

21. Akin FW, Murnane OD. Vestibular evoked myogenic potentials. In: Jacobson GP, Shepard NT, eds. Balance function assessment and management. San Diego; Oxford; Brisbane: Plural Publishing; 2008:405-34

22. Teggi R, Ceserani N, Luce FL, Lazzarin A, Bussi M. Otoneurological findings in human immunodeficiency virus positive patients. J Laryngol Otol 2008;122:1289-94 
23. Teggi R, Giordano L, Pistorio V, Bussi M. Vestibular function in HIV patients: preliminary report. Acta Otolaryngol 2006;26:140-6

24. Castello E, Baroni N, Pallestrini E. Neurotological and auditory brain stem response findings in human immunodeficiency virus-positive patients without neurologic manifestations. Ann Otol Rhinol Laryngol 1998;107:1054-60

25. Hausler R, Vibert D, Koralnik IJ, Hirschel B. Neuro-otological manifestations in different stages of HIV infection. Acta Otolaryngol Suppl 1991;481:515-21

26. White JA. Laboratory tests of vestibular and balance functioning. In: Hughes GB, Pensak ML, eds. Clinical otology. Third ed. New York: Thieme; 2007:132-49

27. Young YH. Vestibular evoked myogenic potentials: A clinical tool for testing the inferior vestibular nerve integrity. Acta Neurol Taiwan 2002;11:120-7

28. Ozeki H, Iwasaki S, Ushio M, Takeuchi N, Murofushi T. The lesion site of vestibular dysfunction in Ramsay Hunt syndrome: A study by click and galvanic VEMP. J Vestib Res $2006 ; 16: 217-22$

2. ag Iski . Vestibular-evoked myogenic potentials and caloric stimulation in infants with congenital cytomegalovirus infection. J Laryngol Otol 2008;122:574-9

30. Meynard JL, El Amrani M, Meyohas MC, Fligny I, Gozlan J, Rozenbaum W, et al. Two cases of cytomegalovirus infection revealed by hearing loss in HIV- infected patients. Biomed Pharmacother. 1997;51:461-3

31. Vancíková Z, Dvorák P. Cytomegalovirus infection in immunocompetent and immunocompromised individuals--a review. Curr Drug Targets Immune Endocr Metabol Disord 2001;1:179-87 
32. Matas CG, Silva SM, de Almeida Marcon B, Gonçalves IC. Electrophysiological manifestations in adults with HIVIAIDS submitted and not submitted to antiretroviral therapy. Pro-Fono 2010;22:107-12

33. Moyle G. Clinical manifestations and management of antiretroviral nucleoside analogrelated mitochondrial toxicity. Clin Ther 2000;22:911-36

34. Warnke D, Barreto J, Temesgen Z. Therapeutic review: Antiretroviral drugs. J Clin Pharmacol 2007;47:1570-9

35. Simdon J, Watters D, Bartlett S, Connick E. Ototoxicity associated with use of nucleoside analog reverse transcriptase inhibitors: A report of 3 possible cases and review of the literature. Clin Infect Dis 2001;32:1623-7

36. Cohen HS, Cox C, Springer G, Hoffman HJ, Young MA, Margolick JB, et al. Prevalence of abnormalities in vestibular function and balance among HIV-seropositive and HIV-seronegative women and men. PLOS ONE. 2012;7:1-7

37. Reyes-Contreras L, Silva-Rojas A, Ysunza-Rivera A, Jiménez-Ruíz G, Berruecos-Villalobos P, Romo-Gutiérrez G. Brainstem auditory evoked response in HIV-infected patients with and without AIDS. Arch Med Res 2002;33:25-8

38. Bankaitis AE, Keith RW. Audiological changes associated with HIV infection. Ear Nose Throat J 1995;74:353-6+358

39. Dellepiane M, Medicina MC, Mora R, Salami A. Static and dynamic posturography in patients with asymptomatic HIV-1 infection and AIDS. Acta Otorhinolaryngol Ital 2005;25:353-8 


\section{Summary}

- The vestibulocollic reflex (VCR) stabilizes the head during active movements

- Testing of the vestibulo-ocular reflex (VOR) is the basis of many commonly used vestibular tests

- There is a significant high occurrence of abnormal vestibular function in adults infected with HIV, but previous reports concentrated mainly on VOR tests

- It is currently unknown if HIV affects the VCR pathways

- Cervical/colic vestibular evoked myogenic potentials (cVEMPs) allow direct testing of VCR pathways

- Abnormal cVEMP recordings showed a high occurrence of abnormalities of the VCR pathways in adults living with the human immunodeficiency virus (HIV) 